\title{
Symmetry lowering of pentacene molecular states interacting with a $\mathrm{Cu}$ surface
}

\author{
Chiara Baldacchini, * Carlo Mariani, and Maria Grazia Betti \\ Dipartimento di Fisica, Università di Roma La Sapienza, Piazzale A. Moro 2, I-00185 Roma, Italy \\ Ivana Vobornik, Jun Fujii, Emilia Annese, and Giorgio Rossi ${ }^{\dagger}$ \\ INFM-CNR-TASC Laboratory, Area Science Park, S.S. 14, km 163.5, Basovizza, I-34012 Trieste, Italy \\ Andrea Ferretti, Arrigo Calzolari, Rosa Di Felice, Alice Ruini, and Elisa Molinari \\ INFM-CNR-S3, National Center on nanoStructures and bioSystems at Surfaces, I-41100 Modena, Italy \\ and Dipartimento di Fisica, Università di Modena e Reggio Emilia, I-41100 Modena, Italy
}

(Received 30 August 2007; published 26 December 2007)

\begin{abstract}
Pentacene adsorbed on the $\mathrm{Cu}(119)$ vicinal surface forms long-range ordered chain structures. Photoemission spectroscopy measurements and $a b$ initio density functional theory simulations provide consistent evidences that pentacene molecular orbitals mix with the copper bands, giving rise to interaction states localized at the interface. Angular-resolved and polarization dependent photoemission spectroscopy shows that most of the pentacene derived intensity is strongly dichroic. The symmetry of the molecular states of the free pentacene molecules is reduced upon adsorption on $\mathrm{Cu}(119)$, as a consequence of the molecule-metal interaction. Theoretical results show a redistribution of the charge density in $\pi$ molecular states close to the Fermi level, consistent with the photoemission intensities (density of states) and polarization dependence (orbital symmetry).
\end{abstract}

DOI: 10.1103/PhysRevB.76.245430

PACS number(s): 73.20.Hb, 68.43.Bc, 79.60.Dp

\section{INTRODUCTION}

The widespread interest in the application of electronically functional organic materials has revived the study of fundamental properties of $\pi$-conjugated molecular systems and hybrid interfaces. ${ }^{1-4}$ Electronic, optical, and transport properties of simple $\pi$-conjugated organic systems deposited on suitable substrates can be tailored by modifying the molecule-molecule interaction and the molecule-substrate coupling. ${ }^{2}$

The molecular ordering on a surface is ruled by a delicate balance between intermolecular forces and substratemolecule interactions. These interactions can be controlled to some extent by the appropriate choice of substrate material and crystallographic symmetry. Pentacene $\left(\mathrm{C}_{22} \mathrm{H}_{14}\right)$ molecules in molecular solids or in ultrathin films deposited on surfaces are typically bound by van der Waals interactions. In spite of high molecular mobility, the pentacene molecules deposited on suitable metallic substrates display long-rangeordered structures. ${ }^{2,5-10}$ Long pentacene chains are found to align along $\mathrm{Cu}$ step edges ${ }^{8}$ or along the Au surface reconstructed channels, ${ }^{7,11}$ as a consequence of the non-negligible molecule-substrate interactions, which may, in fact, drastically influence the molecular self-organization in the film growth. ${ }^{7}$ However, the nature of the interaction between planar $\pi$-conjugated organic molecules and noble metal surfaces is still under debate. ${ }^{2,12-18}$ Recently, experimental evidences of electronic state hybridization between benzene and polyacene molecules and noble metal surfaces have been reported, ${ }^{12,19-21}$ and theoretical investigations predicted the modification of molecular orbitals in pentacene adsorbed on noble metal surfaces. ${ }^{12,22}$

In this paper, we report our study of pentacene on copper, based on experiments and theoretical analysis, addressing the electronic mixing between pentacene molecules [Fig. 1(a)] and the substrate ${ }^{12}$ and the orbital symmetries at the interface. We study the long-range-ordered interface formed by pentacene molecules on the $\mathrm{Cu}(119)$ vicinal surface, whose atomic geometry and growth morphology have been extensively characterized. $7,8,12,20,23-25$ The electronic spectral density is measured by means of synchrotron-based highresolution angular-resolved UV photoemission spectroscopy (HR-ARUPS) with polarized radiation. The electronic structure and the density of states (DOS) are computed from first principles in the framework of density functional theory (DFT) for a model system that fairly mimics the experimental target. The experimental and theoretical studies concur to provide a unified picture of the interface electronic states. Some of us have recently provided clear evidence that hybridization occurs between pentacene molecular orbitals and copper electronic states in this system, and new electronic features emerge in the valence band energy range close to the Fermi level and to the metal $d$ edge. ${ }^{12}$ Here, we report on the formation of hybrid electronic states in a more extended energy range between the Fermi level and a binding energy (BE) of $5.5 \mathrm{eV}$. We also discuss the symmetry of the ob-

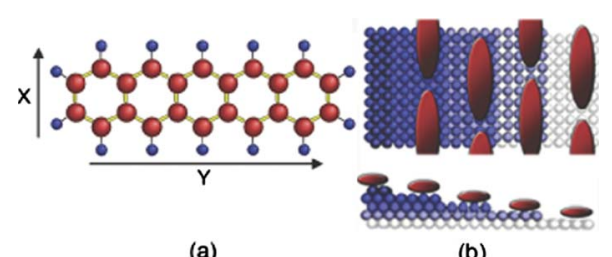

FIG. 1. (Color online) (a) Pentacene molecule with the symmetry directions as labeled in the text. (b) Sketch of pentacene molecules aligned along the $\mathrm{Cu}(119)$ step edges, as deduced by scanning tunneling microscopy (Ref. 8). 


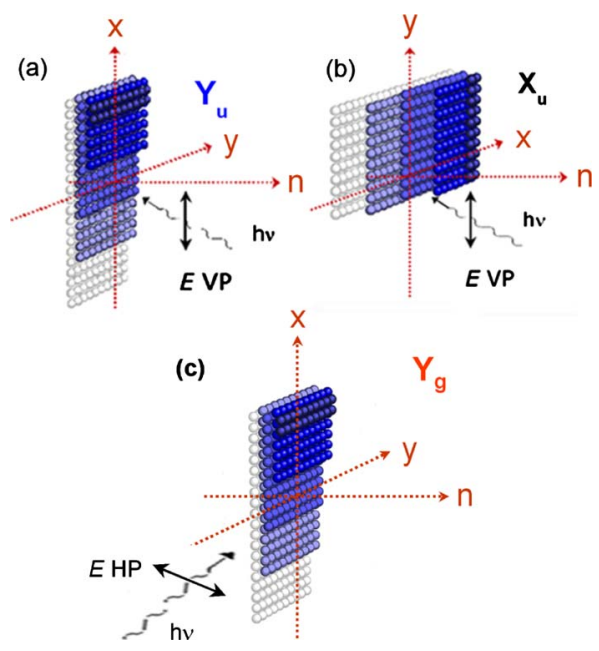

FIG. 2. (Color online) Geometry of the photoemission experiments with polarized light. (a) Vertical polarized light with electric field vector perpendicular to the step edges at normal emission $\left(\mathrm{VP}_{\perp}\right)$; in this configuration, the ${ }^{p} Y$ plane coincides with the scattering plane and $Y_{u}$ states are detected. (b) Vertical polarized light with electric field parallel to the step edges at normal emission $\left(\mathrm{VP}_{\|}\right)$; in this configuration, the ${ }^{p} X$ plane coincides with the scattering plane and $X_{u}$ states are detected. (c) Horizontal polarized (HP) light; in this configuration, $Y_{g}$ states are detected.

served interface states as deduced by using polarized light, by exploiting the high anisotropy of the molecular superstructures, and by performing a symmetry-based analysis of the theoretical results.

\section{EXPERIMENTAL AND COMPUTATIONAL DETAILS}

\section{A. Sample preparation}

$\mathrm{Cu}(119)$ is a miscut single crystal $\left[8.9^{\circ}\right.$ off the (001) low Miller-index surface] with the vicinal clean surface obtained after several cycles of sputtering $\left(\mathrm{Ar}^{+}, E=600 \mathrm{eV}\right)$ and annealing at $740 \mathrm{~K}$ followed by slow cooling to room temperature. This procedure leads to the proper step reconstruction and the long-range order of the stepped structure, as deduced by the spot splitting observed in the low energy electron diffraction (LEED) pattern. ${ }^{7,8}$ Pentacene was deposited in situ by exposing the substrate to the organic molecular beam from a resistively heated quartz crucible, at a rate of $1 \AA / \mathrm{min}$.

\section{B. Photoemission setup}

The HR-ARUPS experiments have been performed at the APE-INFM beamline on the Elettra storage ring in Trieste. Radiation in the energy range $10-100 \mathrm{eV}$ is generated by an AppleII-type quasiperiodic undulator that allows full polarization control and high spectral purity. In this study, we exploited linearly polarized light with horizontal polarization (HP) or vertical polarization (VP) vector (see Fig. 2) with respect to the scattering plane that contains the sample surface normal. The light is monochromatized by a variable spacing grating and focused on the sample into a spot of $\sim 150 \times 50(H \times V) \mu \mathrm{m}^{2}$. The emitted photoelectrons are detected by a hemispherical analyzer Scienta SES 2002, operating at energy resolution $<20 \mathrm{meV}$ and angular resolution of $0.2^{\circ}$.

The normal emission condition is hereafter defined with respect to the (001) terraces rather than for the (119) vicinal surface. Figure 2 shows the geometrical arrangement of the ARUPS measurements reported here: the scattering plane, defined by the surface normal and the incoming photon direction, was fixed and the light polarization vector could be oriented with respect to it. Also, the polar and azimuthal angles of the sample could be oriented with respect to the scattering plane, in order to exploit the selection rules on the parity of the initial electronic states excited by the photoemission process. The HP radiation was used at a grazing incidence angle of $12^{\circ}$ and the sample azimuth was chosen to expose the step edges horizontally, i.e., parallel to the polarization vector [Fig. 2(c)]. The VP radiation was used at $45^{\circ}$ incidence angle, with the sample azimuth such to expose the step edges perpendicular $\left[\mathrm{VP}_{\perp}\right.$, Fig. $\left.2(\mathrm{a})\right]$ or parallel $\left[\mathrm{VP}_{\|}\right.$, Fig. 2(b)] to the polarization vector.

An electric field lying parallel to the sample terraces (VP) allows at any polar angle the excitation of photoelectrons coming from initial states antisymmetric with respect to the scattering plane. With the assignment of the $Y(X)$ long (short) molecular axis given in Fig. 1(a), let us define the corresponding ${ }^{p} Y\left({ }^{p} X\right)$ plane perpendicular to the molecular plane and containing the $Y(X)$ axis. Thus, with such definitions, only electronic states $Y_{u}\left(X_{u}\right)$, antisymmetric for reflection with respect to the ${ }^{p} Y\left({ }^{p} X\right)$ plane, are detectable when using $\mathrm{VP}_{\perp}\left(\mathrm{VP}_{\|}\right)$radiation [Figs. 2(a) and 2(b)]. Instead, initial states that are $Y_{g}$ symmetric with respect to the scattering plane can be excited by HP radiation, i.e., when the electric field vector has a component perpendicular to the surface.

\section{Computational setup}

Numerical simulations are based on DFT implemented using plane waves and ultrasoft pseudopotentials. The PWSCF package $^{26}$ is used. The simulation cell includes five planes of a $4 \times 7$ slab of $\mathrm{Cu}(100)$ and one molecule. In the chosen initial condition for a structural relaxation, a pentacene molecule is placed above the surface with the major axis parallel to the step edges as in Fig. 1, with its center on top of the fourfold hollow site. Full relaxation of the ionic positions is performed until the forces acting on the atoms are smaller than $26 \mathrm{meV} / \AA$. A $4 \times 2$ grid of $\mathbf{k}$ points is adopted to sample the surface Brillouin zone (SBZ) and a kinetic energy cutoff of $25 \mathrm{Ry}$ (200 Ry) is used for expanding the wave functions (charge densities). The PW91-generalized gradient approximation exchange-correlation functional ${ }^{27}$ is adopted for all the calculations. Further details have been published elsewhere. $^{12}$

Focusing on the relaxed structure, the density of states is computed from the single-particle Kohn-Sham eigenvalues. To highlight molecule-related features, we also compute the DOS projected (pDOS) onto the eigenstates $\left|\phi_{i}\right\rangle$ of the isolated pentacene molecule, 


$$
\operatorname{pDOS}_{i}(\omega)=\frac{1}{N_{\mathbf{k}} \sum_{n}}\left|\left\langle\phi_{i} \mid \psi_{n \mathbf{k}}\right\rangle\right|^{2} \delta\left(\omega-\epsilon_{n \mathbf{k}}\right),
$$

where $\mathbf{k}$ points are summed over the whole $\mathrm{SBZ}, n$ is a band index, and $\epsilon_{n \mathbf{k}}\left(\left|\psi_{n \mathbf{k}}\right\rangle\right)$ are the eigenvalues (eigenvectors) of the interface. Dirac delta functions are replaced with Gaussians (spread of $0.01 \mathrm{Ry}$ ) in actual calculations. Because the molecular states have a well defined symmetry for reflections with respect to the ${ }^{p} X$ and ${ }^{p} Y$ planes, we can define a pDOS function with defined symmetry as well. If $\hat{P}$ is a parity operator having two eigenvalues $P_{u}$ and $P_{g}$,

$$
\operatorname{pDOS}_{P_{u, g}}(\omega)=\sum_{i \in\left\{P_{u, g}\right\}} \operatorname{pDOS}_{i}(\omega)
$$

where the sum is only over the $i$ states having the proper $P_{u}$ or $P_{g}$ symmetry. This is because $\sum_{i \in\left\{P_{u, g}\right\}}\left|\phi_{i}\right\rangle\left\langle\phi_{i}\right|$ is the projector over the $P_{u, g}$ manifold. These latter pDOS functions computed for different parities provide a set of theoretical results that concur with the polarized-light photoemission results in the analysis of the interface states at the pentacene/ copper system.

\section{PHOTOEMISSION RESULTS}

\section{A. Pentacene molecular states}

The photoemission spectrum of a pentacene thick film $(\sim 100 \AA)$ grown on the $\mathrm{Cu}(119)$ vicinal surface kept at room temperature is compared with the photoemission spectral density of pentacene free molecules, as measured in the gas phase (Fig. 3). The $\pi$-molecular orbitals, clearly identified in the gas-phase spectra according to the literature,$^{23,28,29}$ have a one-to-one correspondence with the electronic features observed for the pentacene film. The ionization potential energies of the gas-phase molecular orbitals and the binding energies of the electronic states of the condensed pentacene film are reported in Table I. We also list in Table I the symmetries of the associated wave functions, as obtained by our DFT calculations for an isolated pentacene molecule. Besides the standard notation for the $D_{2 h}$ group (column 1), the symmetries of the pentacene molecular orbitals are also defined (column 4) in terms of reflections with respect to the planes ${ }^{p} X,{ }^{p} Y$ [Fig. 1(a) and Sec. II]. We infer the reflection symmetries from the squared moduli of the calculated molecular states, which are shown in the bottom panel of Fig. 3: a node in the ${ }^{p} X\left({ }^{p} Y\right)$ plane means ungerade symmetry with respect to that plane, indicated as $X_{u}\left(Y_{u}\right)$; the absence of a node means gerade symmetry, indicated as $X_{g}\left(Y_{g}\right)$.

Photoemission data on the pentacene thick film do not present relevant differences when photoelectrons are excited with VP or HP radiation because at $100 \AA$ thickness, the film is polycrystalline. From theoretical results, the $3 b_{2 g}$ highest occupied molecular orbital (HOMO), the $2 b_{2 g}$ and the $1 b_{2 g}$ molecular orbitals are antisymetric with respect to $Y$ and symmetric with respect to $X\left(X_{g} Y_{u}\right)$. The $3 b_{1 g}$ molecular orbital is antisymetric with respect to $X$ and symmetric with respect to $Y\left(X_{u} Y_{g}\right)$. The $2 a_{u}$ and the $1 a_{u}$ states are fully antisymmetric $\left(X_{u} Y_{u}\right)$, while the $3 b_{1 u}$ molecular orbital is totally symmetric $\left(X_{g} Y_{g}\right)$. The lowest unoccupied molecular

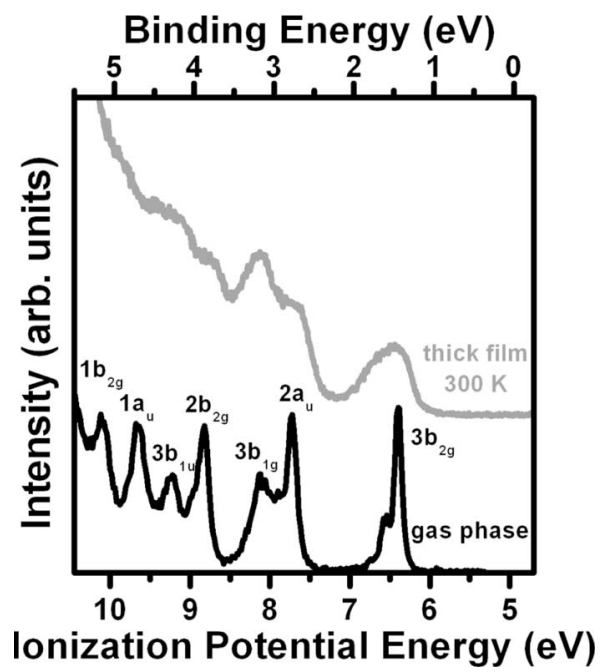

(a)

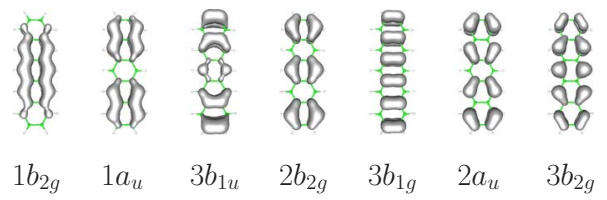

(b)

FIG. 3. (Color online) (a) Dark solid line: photoemission spectrum $(h \nu=21.0 \mathrm{eV})$ of pentacene molecules in the gas phase, with the molecular-orbital peaks labeled according to the $D_{2 h}$ symmetry group (Refs. 28 and 29). Light solid line: photoemission spectrum $(h \nu=18.0 \mathrm{eV})$ of a $100 \AA$ thick pentacene film grown at room temperature on the $\mathrm{Cu}(119)$ surface. The spectra have been aligned to the HOMO $3 b_{2 g}$ peak (Ref. 23). (b) Computed occupied molecular orbitals of pentacene labeled according to their symmetries.

orbital (LUMO), not displayed in Fig. 3, has an $X_{g} Y_{g}$ character.

The results presented in this subsection essentially demonstrate the electronic equivalence between the free gasphase pentacene molecules and the molecules crystallized in

TABLE I. Experimental binding energies (BEs) and ionization potentials (IPs) of occupied molecular orbitals of pentacene in the gas and solid phases, as derived from the photoemission spectra shown in Fig. 3. The HOMO is the $3 b_{2 g}$ state. The rightmost column reports the orbital symmetries from DFT calculations.

\begin{tabular}{lccc}
\hline \hline & $\begin{array}{c}\text { Gas-phase } \\
\text { UPS }\end{array}$ & $\begin{array}{c}\text { Solid-phase } \\
\text { UPS }\end{array}$ & \\
& IP & BE & Symmetry \\
Orbitals & $(\mathrm{eV})$ & $(\mathrm{eV})$ & DFT \\
\hline $3 b_{2 g}$ & 6.40 & 1.50 & $X_{g} Y_{u}$ \\
$2 a_{u}$ & 7.10 & 2.70 & $X_{u} Y_{u}$ \\
$3 b_{1 g}$ & 8.12 & 3.17 & $X_{u} Y_{g}$ \\
$2 b_{2 g}$ & 8.83 & 3.76 & $X_{g} Y_{u}$ \\
$3 b_{1 u}$ & 9.24 & 4.15 & $X_{g} Y_{g}$ \\
$1 a_{u}$ & 9.64 & 4.45 & $X_{u} Y_{u}$ \\
$1 b_{2 g}$ & 10.10 & 4.48 & $X_{g} Y_{u}$ \\
\hline \hline
\end{tabular}




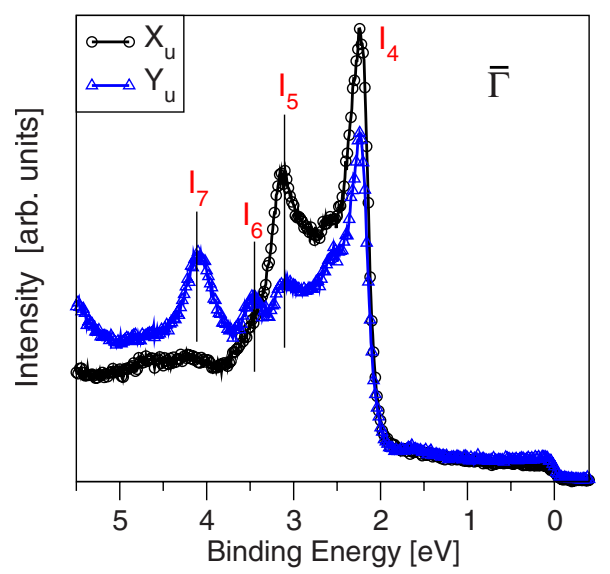

FIG. 4. (Color online) Photoemission spectra $(h \nu=18.0 \mathrm{eV})$ integrated $11.6^{\circ}$ around the $\bar{\Gamma}$ point for the $(3 \times 7)$-pentacene $/ \mathrm{Cu}(119)$ interface prepared at $370 \mathrm{~K}$ and measured at RT. Data collected with $\mathrm{VP}_{\|}\left(\mathrm{VP}_{\perp}\right)$ light are shown with circles (triangles). Due to selection rules in the chosen measurement geometry, the observed electronic features with such polarizations are due to antisymmetric states $X_{u}\left(Y_{u}\right)$ with respect to the ${ }^{p} X\left({ }^{p} Y\right)$ reflection plane. Interface features $I_{4}$ to $I_{7}$ are shown.

a deposited thick film. They constitute the reference situation to understand the electronic modifications that occur at the pentacene/ $\mathrm{Cu}$ interface. The latter can be inspected by measuring the photoemission spectra of a single layer of pentacene on the metal surface and are presented in the next subsection.

\section{B. Pentacene interacting states}

A single layer of pentacene on the $\mathrm{Cu}(119)$ surface forms highly ordered pentacene chains that are aligned along the step edges with an interchain molecule-molecule distance commensurate with the terrace width, as observed by scanning tunneling microscopy (STM) images. ${ }^{8}$ A cartoon of the molecular topography on this surface is depicted in Fig. 1(b). A more packed phase is achieved by increasing the coverage up to the saturation of the molecular adsorption on the $\mathrm{Cu}(119)$ surface kept at about $370 \mathrm{~K}$. This phase is formed by molecular chains lined up along the step edges, commensurate with the surface unit cell, showing a $(3 \times 7)$ longrange-ordered periodicity in the LEED pattern. ${ }^{7,24}$ Previous experimental results and theoretical predictions demonstrate that the pentacene adsorption in the $(3 \times 7)$ phase induces a suppression of the $\mathrm{Cu}$ surface states and the presence of interface states due to the molecule-metal interaction., ${ }^{72,25}$ Identifying the symmetry of the pentacene induced interaction states is possible by exploiting the polarization dependent selection rules of photoemission on the highly ordered $(3 \times 7)$-pentacene superstructure.

Angular-integrated $\left(11.6^{\circ}\right.$ around $\left.\bar{\Gamma}\right)$ photoemission spectra at normal emission of the $(3 \times 7)$-pentacene/ $\mathrm{Cu}(119)$ interface are reported in Fig. 4. These spectra were collected by using VP light with polarization vector parallel or perpendicular to the step edges of the vicinal surface. For a closer

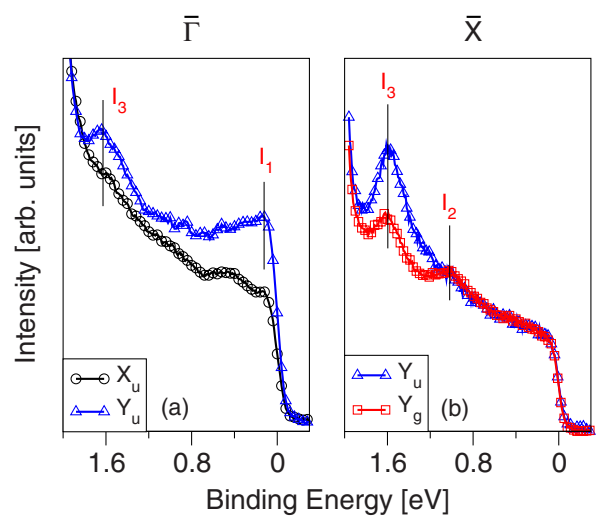

FIG. 5. (Color online) (a) VP spectra $(h \nu=18.0 \mathrm{eV})$ integrated $11.6^{\circ}$ around the $\bar{\Gamma}$ point of the SBZ in the low binding-energy region. The signal plotted with a light line and triangles (dark line and circles) was measured with $\mathrm{VP}_{\perp}\left(\mathrm{VP}_{\|}\right)$radiation and reveals only interface features that derive from $Y_{u}\left(X_{u}\right)$ molecular orbitals. (b) Photoemission spectra $(h \nu=18.0 \mathrm{eV})$ integrated $11.6^{\circ}$ around the $\bar{X}$ point of the $\mathrm{SBZ}$ by using $\mathrm{VP}_{\perp}$ radiation (triangles) and $\mathrm{HP}$ radiation (squares). Given the symmetry explanation of Sec. II, the $\mathrm{VP}_{\perp}$ (HP) spectrum reveals interface states that derive from molecular orbitals of $Y_{u}\left(Y_{g}\right)$ symmetry.

inspection of the low binding-energy region, Fig. 5 displays photoemission spectra in the energy range close to the Fermi level, as obtained with the VP and HP orientations and angle integrated $\left(11.6^{\circ}\right)$ around (a) $\bar{\Gamma}$ and (b) $\bar{X}$. The interface spectral features are labeled as $I_{n}$ ( $n$ index increasing with increasing $\mathrm{BE}$ ): most of them present a strong dichroism.

The $\mathrm{VP}_{\perp}$ (triangles) photoemission data show an enhanced DOS close to the Fermi energy, relative to the $\mathrm{VP}_{\|}$ signal (circles). This enhancement reveals the presence of an electronic state $I_{1}$, located at about $0.15 \mathrm{eV} \mathrm{BE}$ and possessing a $Y_{u}$ reflection symmetry. Another $Y_{u}$ state $I_{3}$, at about $1.5 \mathrm{eV} \mathrm{BE}$, is identified in the same $\mathrm{VP}_{\perp}$ spectrum of Fig. 5. The state $I_{3}$ persists at higher pentacene coverage and in the thick film (see Fig. 3), where it is identified with the $3 b_{2 g}$ HOMO, whose $X_{g} Y_{u}$ symmetry is well established. ${ }^{28,29}$ The symmetry of the HOMO is confirmed in the spectrum collected at the $\bar{X}$ point of the SBZ [Fig. 5(b)]. In fact, it is sharp in the $\mathrm{VP}_{\perp}$ signal (triangles) that detects the $Y_{u}$ symmetry component but quenched in the HP signal (squares) that detects the gerade component only for reflections relative to the ${ }^{p} Y$ plane $\left(Y_{g}\right)$ and not for those relative to the ${ }^{p} X$ plane $\left(X_{g}\right)$. At $\bar{X}$, the state $I_{2}$ is clearly observed using HP light, while it is absent in the $\mathrm{VP}_{\perp}$ spectra. Thus, it has a definite symmetric character with respect to the $Y$ axis of the molecule $\left(Y_{g}\right)$. We did not collect spectra in the proper geometry for directly probing the symmetry of $I_{2}$ with respect to $X$. However, being it not observed when using vertical polarized light in any experimental geometry (both $\mathrm{VP}_{\perp}$ and $\mathrm{VP}_{\|}$), any ungerade symmetry should be excluded and therefore a symmetric character with respect to the $X$ molecular axis can be expected $\left(X_{g}\right)$, as reported in Table II, where the measured BEs and symmetries of the electronic states of the $(3 \times 7)$-pentacene/Cu(119) interface are summarized.

In the $\mathrm{BE}$ region where the $\mathrm{Cu} d$ bands dominate the spectral density, the pentacene induced states cannot be clearly 
TABLE II. Binding energies and symmetries of the interface electronic states of the $(3 \times 7)$-pentacene/Cu(119) interface, as derived from the photoemission spectra shown in Figs. 4 and 5.

\begin{tabular}{lcc}
\hline \hline $\begin{array}{l}\text { Interface states } \\
\text { (Expt.) }\end{array}$ & $\begin{array}{c}\text { Binding energy } \\
(\mathrm{eV})\end{array}$ & Symmetry \\
\hline$I_{1}$ & 0.15 & $Y_{u}$ \\
$I_{2}$ & 1.00 & $X_{g} Y_{g}$ \\
$I_{3}$ & 1.50 & $X_{g} Y_{u}$ \\
$I_{4}$ & 2.25 & $X_{u} Y_{u}$ \\
$I_{5}$ & 3.12 & $X_{u} Y_{g}$ \\
$I_{6}$ & 3.48 & $Y_{u}$ \\
$I_{7}$ & 4.12 & $X_{g} Y_{u}$ \\
\hline \hline
\end{tabular}

identified as in the low binding-energy region. Nonetheless, the VP photoemission spectra for the clean $\mathrm{Cu}(119)$ surface do not present any step-related dichroism of the spectral density; thus, the dichroic features must be assigned to the interface states. The $I_{4}$ interface state is the most intense structure in both VP spectra (Fig. 4), revealing its $X_{u} Y_{u}$ character antisymmetric with respect to both $X$ and $Y$ molecular axes. The $I_{5}$ state is intense when excited by $\mathrm{VP}_{\|}$light and it is barely detectable under $\mathrm{VP}_{\perp}$ irradiation, i.e., it has an $X_{u} Y_{g}$ character antisymmetric with respect to $X$ and symmetric with respect to $Y$. The $I_{6}$ and $I_{7}$ interface states are intense when excited by $\mathrm{VP}_{\perp}$ light, i.e., they are compatible with a $Y_{u}$ character antisymmetric with respect to the long molecular axis. In the spectrum collected with $\mathrm{VP}_{\|}$light, while the $I_{7}$ state is clearly very weak, compatible with an $X_{g}$ character symmetric with respect to the short molecular axis, the $I_{6}$ state is hidden by the highly intense $I_{5}$ structure, and its symmetry cannot be deduced. When excited with VP light [Figs. 4 and 5(a)], most pentacene-induced states have a higher intensity under $\mathrm{VP}_{\perp}$ irradiation and therefore possess a pronounced $Y_{u}$ symmetry component, except for the $I_{2}$ and $I_{5}$ states.

A comparison between the spectral density of the pentacene/ $\mathrm{Cu}(119)$ interface (this subsection) and the photoemission results for the pentacene thick film (previous subsection) may give further interpretation hints to the previous assignments. The $I_{1}$ and the $I_{2}$ states do not have any counterpart in the photoemission spectrum of solid pentacene and can be ascribed to the pentacene-metal interaction involving the LUMO, as deduced in previous experimental and theoretical studies. ${ }^{12}$ The electron mixing due to the pentacenecopper interaction gives rise to interface states whose symmetry might not be directly related to the symmetry of the original molecular state. Indeed, the $I_{1}$ state has a predominant $Y_{u}$ symmetry component, not reminiscent of the $X_{g} Y_{g}$ character of the parent LUMO. The $I_{2}$ peak is also a descendent of the pentacene LUMO: at odds with $I_{1}, I_{2}$ maintains the original $X_{g} Y_{g}$ reflection symmetry of the LUMO. At increasing BEs, two spectral peaks reveal electron states of the pentacene/ $\mathrm{Cu}(119)$ interface that maintain the binding energies and the symmetries of the parent molecular states: (i) $I_{3}$ is $X_{g} Y_{u}$ and derives from the pentacene $3 b_{2 g}$ HOMO and (ii) $I_{5}$ is $X_{u} Y_{g}$ and derives from the $3 b_{1 g}$ molecular orbital (see Fig. 3). The other peaks $I_{4}, I_{6}$, and $I_{7}$, as well as the previ-

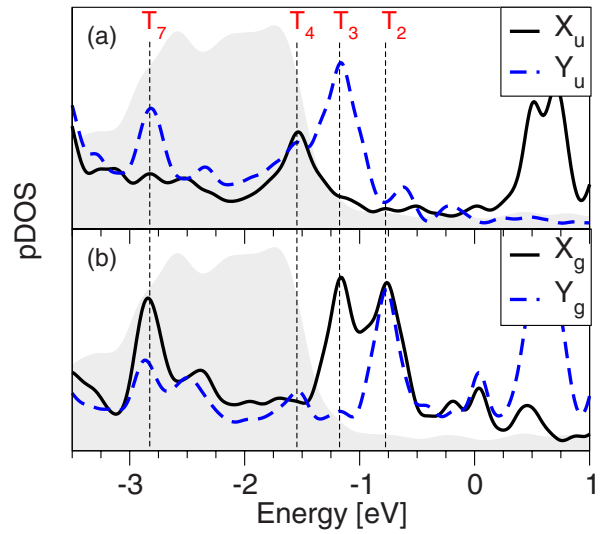

FIG. 6. (Color online) Symmetry-resolved projected density of states; (a) $X_{u}$ (solid line), $Y_{u}$ (dashed line) pDOSs; (b) $X_{g}$ (solid line), $Y_{g}$ (dashed line) pDOSs. As a reference, the total interface DOS (shaded area) is reported (Ref. 12). The assignments of the interface states $T_{2}-T_{4}, T_{7}$ is shown. The Fermi energy is set to zero.

ously mentioned $I_{1}$ and $I_{2}$, do not have a direct correspondence with the parent molecular orbitals due to the symmetry lowering caused by the coupling with the surface. This evidence relative to the orbital symmetries confirms the electronic mixing between pentacene and copper already inferred on the basis of other experimental ${ }^{12,20}$ and theoretical ${ }^{12}$ elements and can be further inspected by a first-principles theoretical analysis of the symmetry-resolved electronic DOS.

\section{THEORETICAL RESULTS}

In Fig. 6, we report the total and molecule-projected DOSs, according to Eqs. (1) and (2). Figures 6(a) and 6(b) show the pDOS curves with defined $(X, Y)_{u}$ and $(X, Y)_{g}$ symmetries, respectively [Eq. (2)]. Around $-0.8 \mathrm{eV}$, we find a clear peak $T_{2}$ with full parity $\left(X_{g} Y_{g}\right)$, which can be attributed to the partial occupation of the pentacene LUMO. This also stems from the LUMO pDOS [Fig. 7(d)]. This state is the theoretical counterpart of the $I_{2}$ feature found experimentally at $1.0 \mathrm{eV} \mathrm{BE}$ and having the same symmetry (see Table II). Moving to lower energies (i.e., higher BEs), we can map the theoretical peak $T_{3}$ at $-1.2 \mathrm{eV}$ with $X_{g} Y_{u}$ symmetry to the $I_{3}$ feature (at $1.5 \mathrm{eV} \mathrm{BE}$ ) due to the hybridization of the molecular HOMO state $\left(3 b_{2 g}\right)$. The $X_{u} Y_{u}$ feature $T_{4}$ with also a slight $Y_{g}$ component at $-1.6 \mathrm{eV}$ displays an important projection onto the HOMO-1 $\left(2 a_{u}\right)$ and HOMO-2 $\left(3 b_{1 u}\right)$ states. We map it to the $I_{4}$ experimental state at $-2.25 \mathrm{eV}$. Being the $\mathrm{Cu}_{d}$ edge at $-1.5 \mathrm{eV}$ theoretically and $2.0 \mathrm{eV} \mathrm{BE}$ experimentally, the $I_{4} / T_{4}$ feature is resonant with the highest $\mathrm{Cu} d$ bands in both pictures. We note that the discrepancy between experimental and DFT Kohn-Sham binding energies increases at higher binding energies. We checked that this discrepancy is almost linearly going to zero as the energy approaches the Fermi level, consistently with the GW corrections found for other systems. ${ }^{30-32}$

At lower energies (higher BE), we identify a clear peak $\left(T_{7}\right)$ in the theoretical DOSs at $-2.8 \mathrm{eV}$ with $X_{g} Y_{u}$ symmetry. The state can be attributed to a mixing of the HOMO $\left(3 b_{2 g}\right)$ 


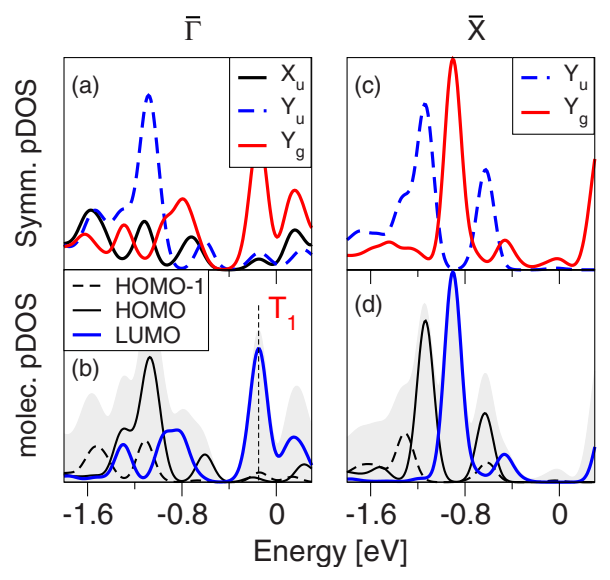

FIG. 7. (Color online) k-point resolved pDOSs. Left panels [(a) and (b)] show pDOSs at the $\bar{\Gamma}$ point in the SBZ, while right panels $\left[(\mathrm{c})\right.$ and (d)] at $\bar{X}$. Upper panels: symmetrized pDOSs $X_{u}$ (dark solid line), $Y_{u}$ (dashed line), and $Y_{g}$ (light solid line). Lower panels: molecular projected pDOS; HOMO-1 $2 a_{u}$ (dashed line), HOMO $3 b_{2 g}$ (thin solid line), and LUMO (thick solid line) states are shown. The $T_{1}$ interface feature is marked. The Fermi energy is set to zero.

and HOMO-2 $\left(3 b_{1 g}\right)$ molecular orbitals on the basis of the pDOSs. Tentatively, we can map $T_{7}$ to the experimental state with the same symmetry $I_{7}$ found around $4.12 \mathrm{eV}$. It would also be possible to relate $T_{7}$ to $I_{6}$ (which has a symmetry at least compatible with $T_{7}$ ) but the binding energy of $I_{6}$ seems to be too close to the theoretical value found for $T_{7}$, according to the just described underestimation of theoretical energies within DFT.

We plot in Figs. 7(a) and 7(c) the symmetrized pDOSs at the $\bar{\Gamma}$ and $\bar{X} \mathbf{k}$ points in the SBZ. This is useful to directly compare with the experimental data in Fig. 5. Further insight is provided by panels (b) and (d) that display the molecular pDOSs in the same energy region. From Fig. 7(a), we see the $Y_{u}$ character related to the $T_{3}(-1.2 \mathrm{eV})$ feature, which has the largest component on the molecular HOMO. The $T_{2}$ peak is clearly evident in Fig. 7(c) around $-0.9 \mathrm{eV}$ in the $Y_{g}$ curve. We see [panel (d)] that this feature is due to the partial occupation of the molecular LUMO state, which is also in agreement with previous results for other conjugated molecules on noble metals. ${ }^{16,18,19}$ This analysis is consistent with the one presented in Fig. 5 for the $I_{2}$ and $I_{3}$ states.

Close to the Fermi level (set to zero) at the $\bar{\Gamma}$ point, we observe a clear peak $T_{1}$ with full parity $\left(X_{g} Y_{g}\right)$ due to the LUMO. This $T_{1}$ feature is found at the same energy and at the same SBZ point as the $I_{1}$ state. On this basis, we believe that the theoretical $T_{1}$ peak can be associated with the experimental $I_{1}$ peak, despite the discrepancy in the detected
TABLE III. Energies and symmetries of the interface electronic states from DFT simulations.

\begin{tabular}{lccc}
\hline \hline $\begin{array}{l}\text { Interface states } \\
\text { (Theor.) }\end{array}$ & $\begin{array}{c}\text { Energy } \\
(\mathrm{eV})\end{array}$ & Symmetry & Expt. mapping \\
\hline$T_{1}$ & 0.20 & $X_{g} Y_{g}$ & $\left(I_{1}\right)$ \\
$T_{2}$ & 0.90 & $X_{g} Y_{g}$ & $I_{2}$ \\
$T_{3}$ & 1.20 & $X_{g} Y_{u}$ & $I_{3}$ \\
$T_{4}$ & 1.60 & $X_{u} Y_{u}$ & $I_{4}$ \\
$T_{7}$ & 2.80 & $X_{g} Y_{u}$ & $I_{7}\left(I_{6}\right)$ \\
\hline \hline
\end{tabular}

symmetry $\left(X_{g} Y_{g}\right.$ for $T_{1}$ and $Y_{u}$ for $\left.I_{1}\right)$. This can be taken as an indirect evidence of the hybrid nature of the electron state $I_{1}$ : in fact, albeit derived from the parent molecular LUMO state, it does acquire a different symmetry due to the mixing with valence charge of the substrate [see Fig. 2(b) of Ref. 12]. The theoretical features related to the interface are summarized in Table III.

\section{CONCLUSIONS}

We studied the interface electronic properties of a flat long-range-ordered pentacene layer adsorbed on the low index terraces of a vicinal surface of copper by means of angular-resolved photoemission experiments and DFT simulations. Concurrent experimental and theoretical results on the electronic structure of the pentacene/copper interface give a picture for the interface between the single molecular layer and the metal substrate. Thanks to the polarization dependence of photoemission in various experimental geometries and to the symmetry-based theoretical analysis, we could identify those states that preserve, in the adsorbate phase, the molecular-orbital character and those that, instead, represent the true hybridization with the substrate. The interacting states close to the Fermi energy are understood as arising from the partial occupation of the molecular LUMO definitely hybridized with $\mathrm{Cu}$ valence states. Our findings demonstrate that physisorption is not the correct mechanism to describe this system: instead, a clear molecule-substrate hybridization occurs.

\section{ACKNOWLEDGMENTS}

We thank Carlo Cavazzoni and CINECA for support and computing time. We are grateful to the staff of the APEINFM beamline and to the staff of Elettra for operating the storage ring. Funding was provided by Italian MIUR through PRIN2004 and by Università di Roma La Sapienza. A.C. thanks the "Nanofaber" regional laboratory by Regione Emilia Romagna. 
*Corresponding author; present address: Biophysics \& NanoScience Centre and CNISM, Università della Tuscia, P.le dell'Università, I-01100 Viterbo, Italy; baldacchini@unitus.it

${ }^{\dagger}$ Also at: Dipartimento di Fisica, Università di Modena e Reggio Emilia, I-41100 Modena, Italy.

${ }^{1}$ M. Eremtchenko, J. A. Schaefer, and F. S. Tautz, Nature (London) 425, 602 (2003).

${ }^{2}$ G. Witte and C. Wöll, J. Mater. Res. 19, 1889 (2004).

${ }^{3}$ T. Heim, K. Lmimouni, and D. Vuillaume, Nano Lett. 4, 2145 (2004).

${ }^{4}$ H. Kakuta, T. Hirahara, I. Matsuda, T. Nagao, S. Hasegawa, N. Ueno, and K. Sakamoto, Phys. Rev. Lett. 98, 247601 (2007).

${ }^{5}$ V. Corradini, C. Menozzi, M. Cavallini, F. Biscarini, M. G. Betti, and C. Mariani, Surf. Sci. 532-535, 249 (2003).

${ }^{6}$ P. Guaino, D. Carty, G. Hughes, O. McDonald, and A. A. Cafolla, Appl. Phys. Lett. 85, 2777 (2004).

${ }^{7}$ C. Baldacchini, C. Mariani, and M. G. Betti, J. Chem. Phys. 124, 154702 (2006).

${ }^{8}$ L. Gavioli, M. Fanetti, M. Sancrotti, and M. G. Betti, Phys. Rev. B 72, 035458 (2005).

${ }^{9}$ S. Lukas, G. Witte, and C. Wöll, Phys. Rev. Lett. 88, 028301 (2001).

${ }^{10}$ P. G. Schroeder, C. B. France, J. B. Park, and B. A. Parkinson, J. Appl. Phys. 91, 3010 (2002).

${ }^{11}$ L. Floreano, A. Cossaro, D. Cvetko, G. Bavdek, and A. Morgante, J. Phys. Chem. B 110, 4908 (2006).

${ }^{12}$ A. Ferretti, C. Baldacchini, A. Calzolari, R. Di Felice, A. Ruini, E. Molinari, and M. G. Betti, Phys. Rev. Lett. 99, 046802 (2007).

${ }^{13}$ A. Hauschild, K. Karki, B. C. C. Cowie, M. Rohlfing, F. S. Tautz, and M. Sokolowski, Phys. Rev. Lett. 94, 036106 (2005).

${ }^{14}$ K. Lee and J. Yu, Surf. Sci. 589, 8 (2005).

${ }^{15}$ Y. Zou, L. Kilian, A. Schöll, T. Schmidt, R. Fink, and E. Umbach,
Surf. Sci. 600, 1240 (2006).

${ }^{16}$ R. Temirov, S. Soubatch, A. Luican, and F. S. Tautz, Nature (London) 444, 350 (2006).

${ }^{17}$ A. Gerlach, S. Sellner, F. Schreiber, N. Koch, and J. Zegenhagen, Phys. Rev. B 75, 045401 (2007).

${ }^{18}$ A. Alkauskas, A. Baratoff, and C. Bruder, Phys. Rev. B 73, 165408 (2006).

${ }^{19}$ N. Lorente, M. F. G. Hedouin, R. E. Palmer, and M. Persson, Phys. Rev. B 68, 155401 (2003).

${ }^{20}$ C. Baldacchini, F. Allegretti, R. Gunnella, and M. G. Betti, Surf. Sci. 601, 2603 (2007).

${ }^{21}$ D. Käfer and G. Witte, Chem. Phys. Lett. 442, 376 (2007).

${ }^{22}$ K. Lee, J. Yu, and Y. Morikawa, Phys. Rev. B 75, 045402 (2007).

${ }^{23}$ C. Baldacchini, C. Mariani, M. G. Betti, L. Gavioli, M. Fanetti, and M. Sancrotti, Appl. Phys. Lett. 89, 152119 (2006).

${ }^{24}$ L. Gavioli, M. Fanetti, D. Pasca, M. Padovani, M. Sancrotti, and M. G. Betti, Surf. Sci. 566-568, 624 (2004).

${ }^{25}$ C. Baldacchini, M. G. Betti, V. Corradini, and C. Mariani, Surf. Sci. 566-568, 613 (2004).

${ }^{26}$ S. Baroni, A. Dal Corso, S. de Gironcoli, and P. Giannozzi, 2001, http://www.pwscf.org

${ }^{27}$ J. P. Perdew, J. A. Chevary, S. H. Vosko, K. A. Jackson, M. R. Pederson, D. J. Singh, and C. Fiolhais, Phys. Rev. B 46, 6671 (1992).

${ }^{28}$ H. Ozaki, J. Chem. Phys. 113, 6361 (2000).

${ }^{29}$ N. E. Gruhn, D. A. da Silva Filho, T. G. Bill, M. Malagoli, V. Coropceanu, A. Kahn, and J.-L. Brédas, J. Am. Chem. Soc. 124, 7918 (2002).

${ }^{30}$ L. Hedin, Phys. Rev. 139, A796 (1965).

${ }^{31}$ L. Hedin and S. Lundqvist, Solid State Phys. 23, 1 (1969).

${ }^{32}$ A. Marini, R. Del Sole, A. Rubio, and G. Onida, Phys. Rev. B 66, 161104(R) (2002). 